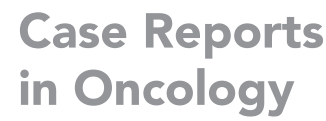

Case Reports

incology

\title{
Alpha-Fetoprotein-Producing Lung Hepatoid Adenocarcinoma with Brain Metastasis Treated with S-1
}

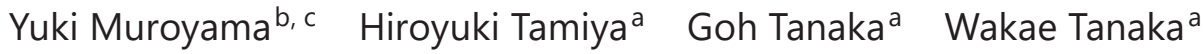 \\ Alexander C. Huangd, e Derek A. Oldridge ${ }^{f}$ Keisuke Matsusakag, h \\ Yutaka Takazawa ${ }^{i}$ Taisuke Jo ${ }^{a, k}$ Tetsuo Ushiku ${ }^{j} \quad T^{2}$ Tahide Nagase ${ }^{a}$ \\ aDepartment of Respiratory Medicine, The University of Tokyo Hospital, Tokyo, Japan; \\ bInstitute for Immunology, Perelman School of Medicine, University of Pennsylvania, \\ Philadelphia, PA, USA; 'Department of System Pharmacology and Translational \\ Therapeutics, Perelman School of Medicine, University of Pennsylvania, Philadelphia, PA, \\ USA; ${ }^{d}$ Department of Medicine, Perelman School of Medicine, University of Pennsylvania, \\ Philadelphia, PA, USA; ${ }^{e}$ Abramson Cancer Center, Perelman School of Medicine, University

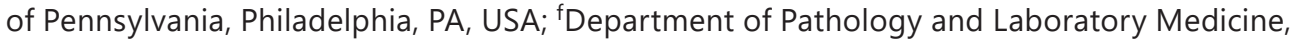 \\ University of Pennsylvania, Philadelphia, PA, USA; 9Department of Molecular Oncology, \\ School of Medicine, Chiba University, Chiba, Japan; hDepartment of Pathology, Chiba \\ University Hospital, Chiba, Japan; 'Department of Pathology, Toranomon Hospital, Tokyo, \\ Japan; jDepartment of Pathology, Graduate School of Medicine, The University of Tokyo, \\ Tokyo, Japan; kDepartment of Health Services Research, The University of Tokyo, Tokyo, \\ Japan
}

\section{Keywords}

Hepatoid adenocarcinoma $\cdot$ Alpha-fetoprotein $\cdot$ Lung $\cdot$ S-1 $\cdot$ 5-Fluorouracil

\begin{abstract}
Lung hepatoid adenocarcinoma (HAC) is a rare primary lung carcinoma pathologically characterized by hepatocellular carcinoma-like tumor cells, the majority of which produce alphafetoprotein (AFP). The clinical prognosis of lung HAC is generally poor, and effective therapeutic regimens for inoperable or recurrent cases have not been established. Here, we report a case of AFP-producing lung HAC with brain metastasis with long-term disease control, treated with the 5-fluorouracil-derived regimen S-1. The patient was a 66 -year-old male admitted to the hospital with alexia. Chest X-ray revealed a massive tumor in the left upper lobe, and a head CT scan revealed a metastasis in the left parietal lobe. The laboratory data showed a remarkably elevated AFP level $(97,561 \mathrm{ng} / \mathrm{mL})$. Pathological assessment of the resected brain tumor revealed HAC, which was compatible with the lung biopsies. Together with the absence
\end{abstract}


of other metastatic lesions, a final diagnosis of primary lung HAC, stage IV T4N3M1b, was given. The patient first underwent non-small cell lung cancer chemotherapy regimens (carboplatin and paclitaxel as the first line, and pemetrexed as the second line), but had clinical progression. After third-line oral S-1 (tegafur/gimeracil/oteracil) administration, the serum AFP level significantly dropped and the patient achieved long-term disease control without relapse, surviving more than 19 months after disease presentation. The autopsy result was consistent with the diagnosis of primary lung $\mathrm{HAC}$, and immunohistochemical staining was AFP+, glypican $3+$, and spalt-like transcription factor $4+$. Here, we report the case of a rare primary lung $\mathrm{HAC}$ with apparent disease control on S-1 therapy, together with a literature review.

\section{Introduction}

Alpha-fetoprotein (AFP)-producing lung cancer is a rare type of primary lung carcinoma, accounting for about $2 \%$ of all lung cancer [1]. Among them, hepatoid adenocarcinoma (HAC) is a major histological type. HAC is characterized by AFP-producing, hepatocellular carcinomalike tumor cells. The most common primary locus is the stomach, but HAC may also arise from other organs of foregut origin, including the pancreas, bladder, and uterus. Lung HAC is also of foregut origin, but the lung is a comparatively rare primary locus, accounting for only $5 \%$ of all HAC $[2,3]$. Lung HAC was first defined pathologically as an AFP-producing tubular or papillary adenocarcinoma with a sheet-like or trabecular proliferation of neoplastic cells and the presence of cells with abundant, eosinophilic cytoplasm and centrally located nuclei in sheet-like or trabecular portions, resembling those of hepatocellular carcinoma cells [2, 3].

One remarkable etiologic feature is that almost all reported cases have occurred in men, especially of middle-to-older ages (from 40 to 70 years) [3-6]. The prognosis is generally poorer than with other types of non-small cell lung carcinoma (NSCLC), presumably because of higher rates of vascular invasion and metastasis [3-6]. Survival from the time point of diagnosis has varied from 4 days to 7 years, and cases with radical surgical resection have had a better prognosis [3-6]. The prognosis for inoperable or recurrent cases of lung HAC is poor [3-6], with no established or effective therapeutic regimens to date.

Here, we report on a patient with inoperative lung HAC with brain metastasis who achieved long-term survival when treated with S-1 following chemoradiotherapy. S-1 (Taiho Pharmaceutical Co. Ltd., Tokyo, Japan), or Teysuno, is an oral fluoropyrimidine anticancer agent that combines tegafur as the effector drug with two modulators: gimeracil, which inhibits 5-fluorouracil (5-FU) degradation, and oteracil potassium, which limits gastrointestinal toxicity by inhibiting prodrug conversion in the gut, at a molar ratio of 1:0.4:1 [7]. We report on this patient with this very rare lung tumor treated with S-1, together with a literature review.

\section{Case Presentation}

The patient was a 66-year-old male who presented with 3-month-long alexia with agraphia and increasing fatigue. His past medical history included chronic hepatitis B, tuberculosis, diabetes, and intestinal perforation. He had a family history of a father with gastric cancer and a mother with an unspecified cancer. He had smoked 30 cigarettes per day for 46 years.

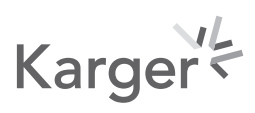




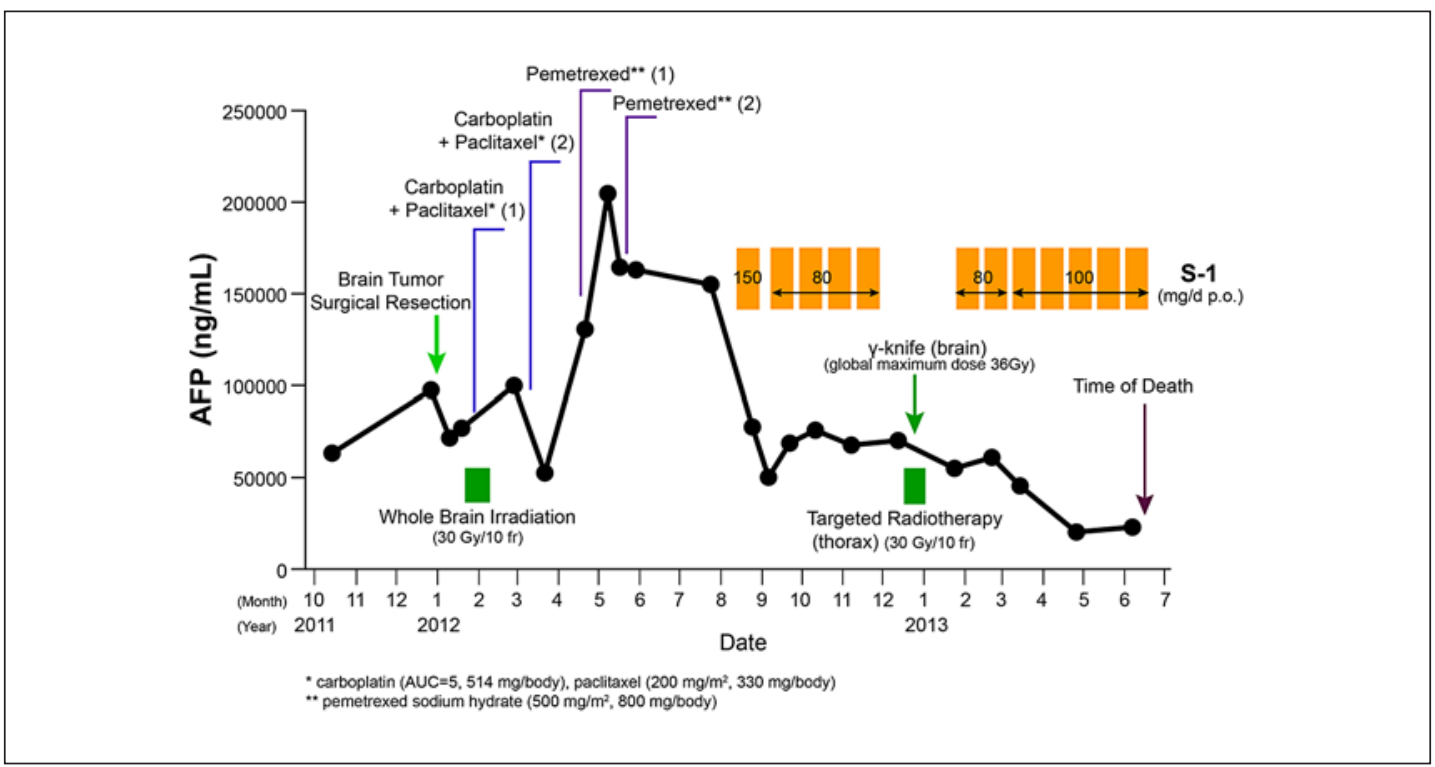

Fig. 1. Clinical course and trend of serum AFP level of the presented case. Kinetics of serum AFP level and timeline of therapies. AFP, alpha-fetoprotein; p.o., per os (by mouth).

At admission, he presented with a slightly decreased level of consciousness. Physical examination revealed decreased left breath sounds and pleural friction rub at the left anterior thorax. There were no palpable lymph nodes. Neurological examination showed impairment of higher brain functions, including alexia with agraphia, a slight component dysfunction, right homonymous hemianopsia, and positive Barré sign. Laboratory data showed a markedly elevated AFP level (97,561 ng/mL; normal range, 0-9) (Fig. 1). Other tumor markers, including carcinoembryonic antigen, beta-human chorionic gonadotropin, and cytokeratin 19 fragment, were slightly above the normal level. Cancer antigen 19-9 and pro-gastrin-releasing peptide levels were within normal limits.

Chest X-ray and chest CT demonstrated a mass $8 \mathrm{~cm}$ in diameter in the anterior segment of the left upper lobe (Fig. 2A, B) with central necrosis. Respiratory-synchronous MRI confirmed that the tumor did not originate from the mediastinum but from the lung itself, as the tumor synchronized with the lung but not with the mediastinum upon respiration (Fig. 2Ca). Chest CT and MRI also revealed tumor embolism that was continuous from the lung tumor, involving the left pulmonary vein, as well as hilar lymph node enlargement (Fig. 2B, $\mathrm{Cb}$ ). Head CT revealed a large tumor $5 \mathrm{~cm}$ in diameter in the left parieto-occipital lobe with surrounding edema and hemorrhage (Fig. 2Da). Contrast MRI showed a ring-enhancing lesion, suggestive of metastatic cancer (Fig. 2Db). An FDG-PET scan revealed distant lymph node metastasis; increased FDG avidity was observed in cervical lymph nodes and splenic hilar lymph nodes, without involvement of other organs. Gastric and colon endoscopy as well as liver and testis ultrasound revealed no additional metastases.

As the patient was neurologically symptomatic, the solitary metastatic brain lesion was surgically resected. The pathological results of the resected tumor showed poorly differentiated hepatoid carcinoma-like cells forming solid, partially adenomatous structures with necrosis. Immunohistochemical stains were highly positive for AFP and glypican 3, highly suggestive of HAC (Fig. 3A). Anti-hepatocyte antibody was weakly positive, yet not conclusive. The lung biopsy specimen showed the same histological pattern (Fig. 3B), and the specimen was also positive for cytokeratin AE1/AE3, suggestive of an epithelial origin. Considering the 


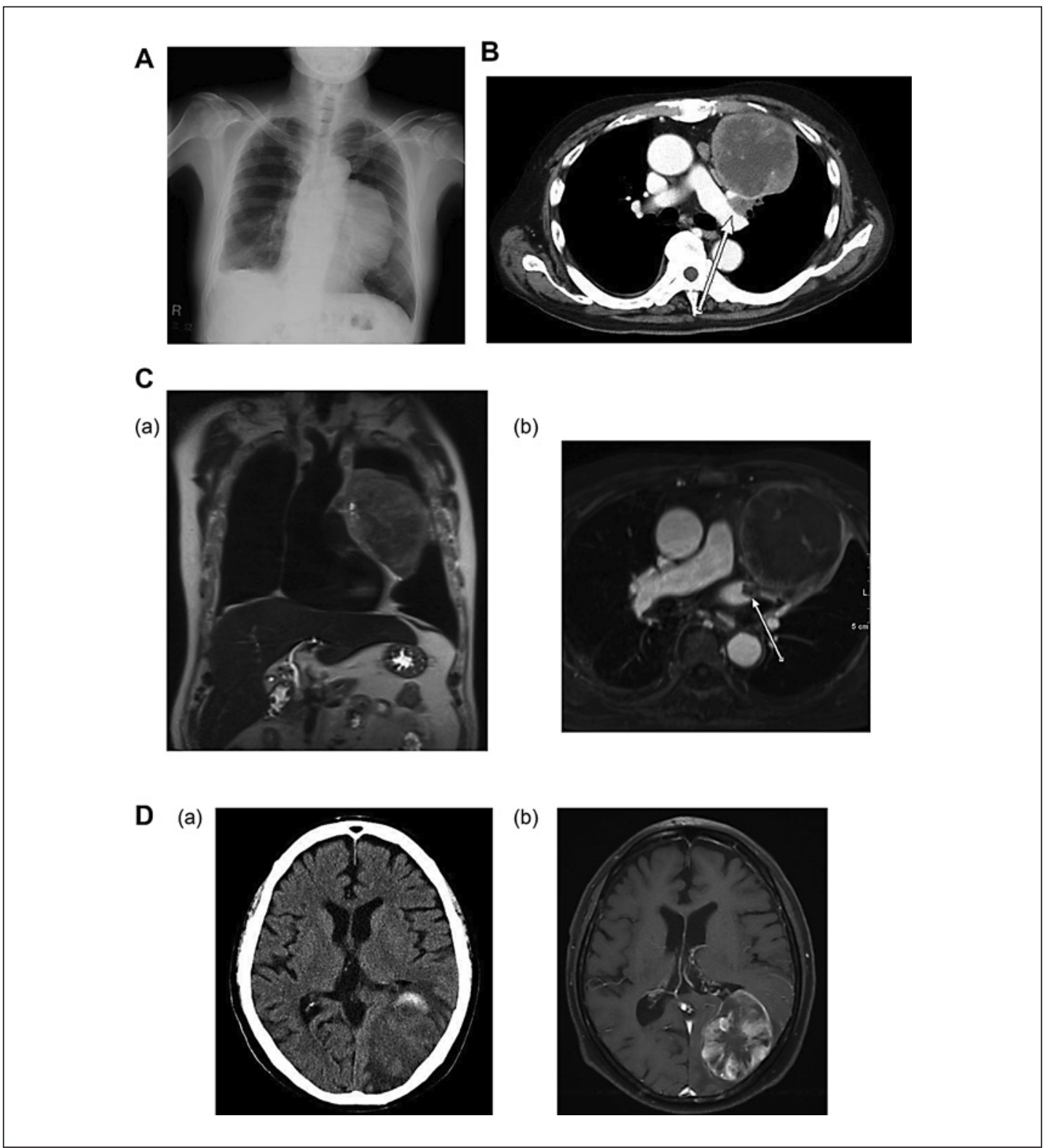

Fig. 2. Imaging studies of the lung hepatoid adenocarcinoma and brain metastasis. A The chest radiographs showed a mass $8 \mathrm{~cm}$ in diameter in the anterior segment of the left upper lobe. B Chest contrast CT, mediastinal window. The chest CT scan revealed a mass in the anterior segment of the left upper lobe with central necrosis. Tumor embolism in the left pulmonary vein (arrow) and enlargement of hilar lymph nodes were also observed. C Respiratory-synchronous MRI (a) confirmed that the tumor did not originate from the mediastinum but from the lung itself, as the tumor synchronized with the lung but not with the mediastinum. Tumor embolism in the left pulmonary vein was observed (b). D Head CT scan (a) and brain MR image (T1W with gadolinium contrast) (b). Head CT showed a large tumor $5 \mathrm{~cm}$ in diameter in the left parieto-occipital lobe with surrounding edema and hemorrhage. Contrast MRI showed a ring-enhancing lesion, suggestive of metastatic cancer. 


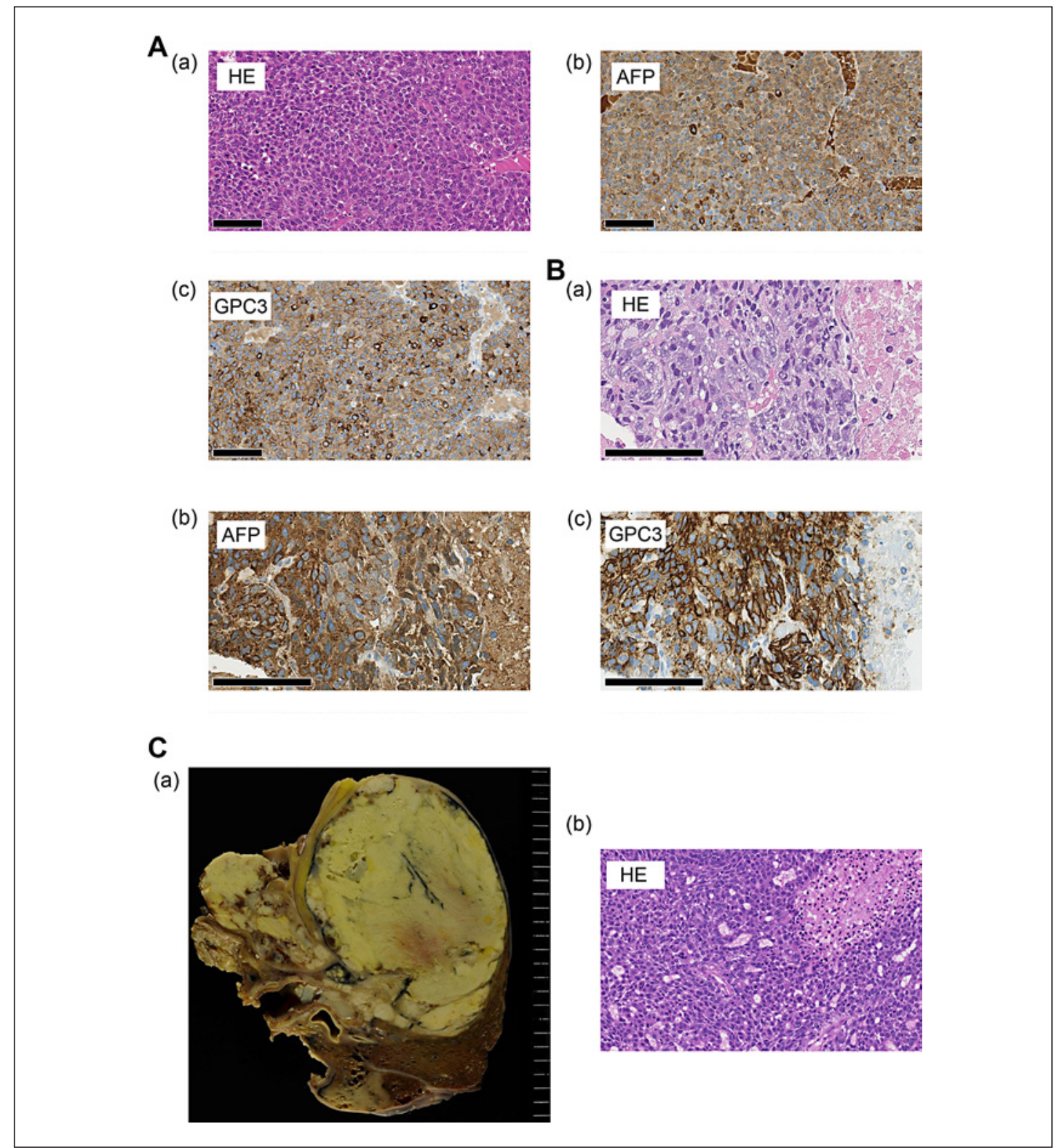

Fig. 3. Pathological characterization of the present case. A The pathology results of the brain metastasis showed poorly differentiated hepatoid cell carcinoma-like cells forming solid structures (a). The immunohistochemical stains were highly positive for AFP (b) and GPC3 (c), all of which are compatible with hepatoid adenocarcinoma. B The pathology results of the lung biopsy showed poorly differentiated hepatoid cell carcinoma-like cells (a). The immunohistochemical stains were highly positive for AFP (b) and GPC3 (c), consistent with the histological characteristics of the metastatic hepatoid adenocarcinoma in the brain. C Lung tumor from the autopsy. a Gross appearance of the lung tumor obtained at autopsy, showing a largely necrotic tumor with invasion to the left pulmonary vein, and with intrusion to the left main bronchus, forming left atrium tumor thrombosis. $\mathbf{b}$ The pathology results of the primary lung tumor showed poorly differentiated hepatoid cell carcinoma-like cells forming solid, partially adenomatous structures with necrosis. Scale bars, $100 \mu \mathrm{m}$. HE, hematoxylin and eosin staining; AFP, alpha-fetoprotein; GPC3, glypican 3. 
absence of lesions in the liver, one of the most common primary organs for AFP-producing tumors, the postoperative diagnosis was determined as lung HAC, stage IV T4N3M1b, based on the TNM Classification of Malignant Tumors, 7th edition.

Postoperative whole-brain irradiation (30 Gy/10 fractions) was performed for residual and/or new metastatic brain tumor. As the primary lung lesion was inoperable, it was treated with chemotherapy. While no standard chemotherapy regimen has been established for lung HAC, one of the recommended first-line regimens for NSCLC is carboplatin and paclitaxel, which was therefore administered as first-line chemotherapy (carboplatin: AUC $=5,514 \mathrm{mg}$ / body; paclitaxel: $200 \mathrm{mg} / \mathrm{m}^{2}, 330 \mathrm{mg} /$ body). Because the patient demonstrated progressive disease, pemetrexed sodium hydrate was administered as the second-line regimen, following NSCLC guidelines (pemetrexed sodium hydrate: $500 \mathrm{mg} / \mathrm{m}^{2}, 800 \mathrm{mg} /$ body). However, the patient continued to progress, according to both imaging study and an elevated AFP level.

With prolonged myelosuppression as a side effect of chemotherapy, oral S-1 was selected as the third-line regimen. After administration of $150 \mathrm{mg} /$ day S-1, the patient's AFP level markedly dropped from about $150,000 \mathrm{ng} / \mathrm{mL}$ to $70,000 \mathrm{ng} / \mathrm{mL}$ (Fig. 1). Concurrent imaging showed a 5.3\% decrease in size of the target lesions (primary tumor, lung metastasis, and mediastinal lymph node metastases), consistent with a stable disease response based on RECIST criteria. As the patient was suffering from persistent nausea and vomiting (grade 3), $\mathrm{S}$-1 was reduced to $80 \mathrm{mg} /$ day for 2-week administration with a 1-week interval. As shown in Figure 1, the patient's AFP level remained stable after introduction of S-1, suggesting that S-1 thwarted the progression of lung HAC. Postoperative recurrent metastatic brain lesions were treated with gamma knife radiosurgery (global max. dose: $36 \mathrm{~Gy}$ ). In addition, thoracic CT revealed an increased size of left atrium tumor thrombosis. Therefore, targeted radiotherapy (30 Gy/10 fractions) was performed to prevent sudden onset of death. The S- 1 dose was increased to $100 \mathrm{mg} /$ day, and the patient was in stable disease control without a remarkable increase in AFP level or size of the primary lung tumor, and he survived for more than 19 months after the diagnosis. The patient died of septic shock from peritonitis due to perforation of the small intestine.

An autopsy was performed, and the tumor workup was consistent with the earlier diagnosis of primary lung HAC with brain metastasis, showing no signs of disease progression. The gross appearance of the lung tumor obtained at autopsy showed a largely necrotic tumor with invasion to the left pulmonary vein, and it also revealed intrusion into the left main bronchus and left atrium tumor thrombosis (Fig. 3Ca). Pathologically, the lung tumor was read as tubular or papillary adenocarcinoma with a sheet-like or trabecular proliferation of neoplastic cells and the presence of cells with abundant, eosinophilic cytoplasm and centrally located, large nuclei, resembling those of hepatocellular carcinoma cells, consistent with lung HAC (Fig. 3Cb). Immunohistochemical staining was positive for AFP, glypican 3 and spalt-like transcription factor 4, partially positive for CD56, and negative for chromogranin A and synaptophysin. Spalt-like transcription factor 4 is an oncofetal protein useful to distinguish hepatic neoplasms and tumors with fetal gut differentiation, and it is only positive in the latter ones [8]. There was no tumor lesion in the intestine, and given the patient's past medical history of jejunum perforation, the cause of death was considered to be independent of HAC, but rather a relapse of the old intestine perforation.

\section{Discussion and Conclusion}

Lung HAC is a rare primary lung carcinoma with a generally poor prognosis, characterized by tumor cells that pathologically resemble hepatocellular carcinoma [3-6]. When examining the literature using the search term "lung, hepatoid adenocarcinoma," with a path- 
ological diagnosis of HAC or at least some hepatoid component, with/without evidence of AFP production (blood AFP elevation and/or immunohistochemical staining for AFP), there are about 60 reported cases of lung HAC [3-6]. Most cases were treated with surgical resection. Contrary to conventional lung carcinoma, there is no established chemotherapy for inoperable cases. Considering its rapid progression and poor prognosis, development of an effective chemotherapy is essential for the treatment of lung HAC.

As far as we have examined, there are approximately 20 reported cases that were treated with chemotherapy, including adjuvant therapy and chemoradiotherapy. Among them, 2 cases were treated with 5-FU [2,9] and 1 case was treated with FK207 (tegafur) [10], a prodrug of 5-FU. All patients presented with stage IV disease with markedly elevated AFP levels $(14,000-320,000 \mathrm{ng} / \mathrm{mL})$, and survival from the time of diagnosis ranged from 6 to 16 months $[2-6,9,10]$. From previous case reports, it is possible that 5 -FU derivatives could be considered an option for advanced HAC with higher AFP levels. The survival time of more than 19 months in the present case is among the longest reported for lung HAC treated with 5 -FU derivatives.

$\mathrm{S}-1$, which we used for the presented case, is an oral combined drug of 5-FU progenitors - tegafur, gimeracil, and oteracil potassium [7]. For gastric HAC, the most common and thus a well-documented HAC, the use of S-1 has been rather common, following practice for gastric cancer treatment, where S-1 is the standard chemotherapy regimen. Despite the existence of many refractory cases, there are several case reports suggesting the effectiveness of S-1 in gastric HAC, showing long-term survival $[11,12]$. As for lung HAC, there are several case reports documenting the efficacy of oral UFT (tegafur/uracil) or S-1 in AFP-producing lung adenocarcinoma, including the report on an 80-year-old man with brain metastasis treated with single-agent UFT with a decrease in AFP level and with significant lung tumor size reduction [13]. There is also a case report of AFP-producing lung adenocarcinoma treated with surgical resection and adjuvant chemotherapy by S-1 with no recurrence [14]. In the aggregate, these reports combined with our own support a general role for S-1 in the treatment of AFP-producing lung cancers.

Here, we reported the case of a rare inoperable AFP-producing primary lung carcinoma with long-term patient survival following treatment with S-1 after conventional chemotherapy for NSCLC. To our knowledge, the survival period of more than 19 months observed in this case is among the longest reported for inoperable lung HAC treated with chemotherapy. As no therapeutic regimens are established for this rare type of primary lung tumor, this case suggests $S-1$ as a potential therapeutic option. Further studies are required to elucidate the pathogenesis and to establish an effective therapy for lung HAC.

\section{Acknowledgments}

We thank the staff of the Department of Respiratory Medicine, the University of Tokyo Hospital, and the Fugaku Trust for Medicinal Research for their support.

\section{Statement of Ethics}

This case report is part of a retrospective study that was approved by the Ethics Board of the Graduate School of Medicine and Faculty of Medicine, The University of Tokyo (approval registration No. 2739). This study uses an opt-out method, in which written informed consent for publication of this case report and any accompanying images was waived.

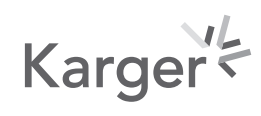




\section{Conflict of Interest Statement}

The authors have no conflicts of interest to declare.

\section{Funding Sources}

This work is supported by a grant-in-aid of the Fugaku Trust for Medicinal Research.

\section{Author Contributions}

Y.M.: study design, writing the manuscript, acquisition and interpretation of data, and review and revision of the manuscript; H.T.: study design, acquisition and interpretation of data, and review and revision of the manuscript; W.T.: acquisition and interpretation of data, and review and revision of the manuscript; G.T.: study design, interpretation of data, and review and revision of the manuscript; K.M. and Y.T.: pathological examination of the biopsy and surgical specimens, acquisition and interpretation of pathological data, and review and revision of the manuscript; T.U.: performing the autopsy, acquisition and interpretation of pathological data, and review and revision of the manuscript; A.C.H. and D.A.O.: data interpretation, and review and revision of the manuscript; T.J.: study supervision, study design, acquisition and interpretation of data, and review and revision of the manuscript; T.N.: study supervision, interpretation of data, and review and revision of the manuscript. All authors approved the final manuscript.

\section{References}

1 Walop W, Chrétien M, Colman NC, Fraser RS, Gilbert F, Hidvegi RS, et al. The use of biomarkers in the prediction of survival in patients with pulmonary carcinoma. Cancer. 1990;65(9):2033-46.

2 Yasunami R, Hashimoto Z, Ogura T, Hirao F, Yamamura Y. Primary lung cancer producing alpha-fetoprotein: a case report. Cancer. 1981;47(5):926-9.

3 Ishikura H, Kanda M, Ito M, Nosaka K, Mizuno K. Hepatoid adenocarcinoma: a distinctive histological subtype of alpha-fetoprotein-producing lung carcinoma. Virchows Arch A Pathol Anat Histopathol. 1990;417(1):73-80.

4 Iino K, Ohta Y, Tamura M, Sato H, Katayanagi K, Kurumaya H. A resected case of alpha-fetoprotein-producing hepatoid adenocarcinoma of the lung. Haigan. 2003;43(1):29-34.

5 Grossman K, Beasley MB, Braman SS. Hepatoid adenocarcinoma of the lung: review of a rare form of lung cancer. Respir Med. 2016;119:175-9.

6 Yang K, Jiang H, Li Q. Primary pulmonary hepatoid adenocarcinoma: a case report and review of the literature. Medicine (Baltimore). 2019;98(14):e15053.

7 Okamoto I, Fukuoka M. S-1: a new oral fluoropyrimidine in the treatment of patients with advanced non-smallcell lung cancer. Clin Lung Cancer. 2009;10(4):290-4.

8 Ushiku T, Shinozaki A, Shibahara J, Iwasaki Y, Tateishi Y, Funata N, et al. SALL4 represents fetal gut differentiation of gastric cancer, and is diagnostically useful in distinguishing hepatoid gastric carcinoma from hepatocellular carcinoma. Am J Surg Pathol. 2010;34(4):533-40.

9 Nasu M, Soma T, Fukushima H, Kudo K, Matsubara O. Hepatoid carcinoma of the lung with production of alphafetoprotein and abnormal prothrombin: an autopsy case report. Mod Pathol. 1997;10(10):1054-8.

10 Hayatsu M, Mori H, Izawa M, Nakazato H, Koishi Y, Yamabe H, et al. Case of alpha-fetoprotein-producing lung cancer with right hemiplegia due to brain metastasis as a main symptom [in Japanese]. Nippon Naika Gakkai Zasshi. 1983;72(8):1035-40.

11 Inagawa S, Shimazaki J, Hori M, Yoshimi F, Adachi S, Kawamoto T, et al. Hepatoid adenocarcinoma of the stomach. Gastric Cancer. 2001;4(1):43-52.

12 Xie Y, Zhao Z, Li P, Wang Y, Guo C, Wang X, et al. Hepatoid adenocarcinoma of the stomach is a special and easily misdiagnosed or missed diagnosed subtype of gastric cancer with poor prognosis but curative for patients of pN0/1: the experience of a single center. Int J Clin Exp Med. 2015;8(5):6762-72.

13 Hirota F, Hosaka K, Funahashi K, Oota M, Ikeda A, Oobayashi Y, et al. Effective treatment of AFP-producing lung cancer with UFT [in Japanese]. Gan To Kagaku Ryoho. 1999;26(3):381-4.

14 Kitada M, Ozawa K, Sato K, Matsuda Y, Hayashi S, Tokusashi Y, et al. Alpha-fetoprotein-producing primary lung carcinoma: a case report. World J Surg Oncol. 2011;9(1):47. 